\title{
Fusion des centres de santé et de services sociaux et bibliothèques : réalités et enjeux au CISSS de Lanaudière
}

\author{
Nancy Gadoury
}

\begin{abstract}
Introduction : La loi 10, entrée en vigueur au Québec le $1^{\text {er }}$ avril 2015, a forcé la fusion des établissements de santé et de services sociaux par région. Les bibliothèques de ces établissements ont été incluses dans la fusion et ont été invitées à revoir leur structure administrative et leur offre de services en fonction de la nouvelle organisation dont ils faisaient désormais partie. Description : Dès avril 2015, les bibliothèques du CISSS de Lanaudière ont entrepris le processus de fusion en développant une mission et une vision adaptée, en intégrant leurs collections, en fusionnant leurs catalogues et en unifiant leurs services. Discussion: Le développement des services en ligne et du travail d'équipe a été primordial pour la réussite du projet de fusion des bibliothèques. Les différentes réalités et enjeux que l'équipe des bibliothèques ont vécus durant la fusion, ainsi que les outils qu'ils ont développés au cours du processus d'intégration sont expliqués à titre d'exemple.
\end{abstract}

\section{Introduction}

La loi 10, modifiant l'organisation et la gouvernance du réseau de santé et de services sociaux au Québec, notamment par l'abolition des agences régionales, est entrée en vigueur le $1^{\mathrm{er}}$ avril 2015 . Elle a forcé une « (...) intégration régionale des services de santé et des services sociaux, la création d'établissements à mission élargie et l'implantation d'une gestion à deux niveaux hiérarchiques, afin de favoriser et de simplifier l'accès aux services pour la population, de contribuer à l'amélioration de la qualité et de la sécurité des soins et d'accroître l'efficience et l'efficacité de ce réseau. » [1]

En résumé, la loi 10 a supprimé un palier de gestion et a réduit le nombre d'établissements à un par région afin d'alléger la structure administrative des services de santé et de services sociaux. L'intégration des soins par région avait également pour but de réduire les dépenses, d'assurer la continuité des soins pour les patients et assurer le partage d'information entre les établissements [2].

Les bibliothèques de santé et de services sociaux ont été incluses dans cette réorganisation administrative, au même titre que les autres départements de soins. Les bibliothèques de santé québécoises ont été invitées, à différents niveaux selon les établissements, à ajuster leur mission, revoir leur structure administrative, leurs méthodes de fonctionnement et leurs offres de services pour répondre aux besoins des nouveaux centres de soins intégrés régionaux.

Cependant, comme tout processus de fusion d'établissements, le chemin était loin d'être tracé d'avance. Plusieurs des établissements québécois fusionnés possédaient leur bibliothèque dont l'identité leur était propre. Certaines de leurs caractéristiques étaient conciliables, d'autres non.

Dans cet article, seront présentées les étapes qu'ont franchies les différentes bibliothèques du Centre intégré de santé et de services sociaux de Lanaudière pour se conformer aux principes instaurés par la Loi 10, qui faisaient d'elles une seule et même entité. Quels sont les enjeux et les réalités que ces bibliothèques ont dû concilier? Et comment a été réalisée cette intégration en pratique?

Tout d'abord, un portrait du nouvel établissement, le CISSS de Lanaudière, sera exposé, ainsi que l'état de situation des différentes bibliothèques au $1^{\mathrm{er}}$ avril 2015. Ensuite, comment, de ce constat des faits, les

Nancy Gadoury. Centre intégré de santé et de services sociaux de Lanaudière, Saint-Charles-Borromée QC

Corresponding author (email: nancy.gadoury@ssss.gouv.qc.ca)

This article has been peer-reviewed.

(c) Gadoury

This article is distributed under a Creative Commons Attribution License: https://creativecommons.org/licenses/by/4.0/ 
bibliothèques en sont venues à réorganiser toutes leurs activités. Plusieurs aspects du projet de fusion des bibliothèques seront discutés: intégration de la structure organisationnelle et administrative, intégration des collections, intégration de l'offre de service et intégration du personnel. En résumé, au cours de 2015, les bibliothèques se sont jointes à différents moments pour répondre aux urgences liées aux fonctionnements et faire l'état de la situation. En 2016, les premières ébauches du projet de fusion des activités ont vu le jour, les premiers mandats ont été donné et les premières actions entamées. Mais c'est uniquement au cours de 2017-2018, soit 2 ans après la fusion officielle, que les projets de fusion ont été réalisés pleinement.

\section{Enjeux et réalités au CISSS de Lanaudière}

«Le CISSS de Lanaudière est le fruit de la fusion de deux centres de santé et de services sociaux (CSSS) de la région, du Centre de réadaptation en déficience physique Le Bouclier, du Centre de réadaptation en déficience intellectuelle La Myriade ainsi que des Centres jeunesse de Lanaudière. Le CISSS de Lanaudière dessert une population de 492234 personnes résidant sur un territoire de $12309 \mathrm{~km}$ carrés, qui connaît une importante croissance démographique. Le CISSS de Lanaudière compte environ 10000 employés et a un budget annuel évalué à environ 850 millions de dollars. » [3]

Le nouveau CISSS de Lanaudière, au $1^{\text {er }}$ avril 2015, comptait 5 bibliothèques, certaines étant fonctionnelles et d'autres moins. Le Tableau 1 (Appendice A) présente un portrait détaillé de ces établissements fusionnés, de leurs bibliothèques et de leurs ressources au $1^{\mathrm{er}}$ avril 2015. Au total, il ne restait que l'équivalent d'un seul poste de bibliothécaire pour tout le CISSS et un peu plus de 3 postes de techniciens en documentation, l'équivalent d'environ 3 postes ayant été coupés dans les mois précédant la fusion. Près de $40 \%$ du budget d'acquisition avait également disparu entre 2013 et 2015 à cause des diverses coupures faites par les établissements avant le $1^{\text {er }}$ avril 2015, l'abolition de l'agence, ainsi que la perte de la bibliothèque du Bouclier maintenant intégrée dans un autre CISSS.

Les 3 bibliothèques qui possédaient du personnel se virent confier la mission de desservir la totalité des établissements fusionnés au $1^{\text {er }}$ avril 2015, ce qui signifiait environ $10 \%$ de hausse de clientèle, avec des ressources partiellement amputées.

\section{Fusion des bibliothèques du CISSS de Lanaudière}

«In a perfect world, undertakings such as mergers, library catalog systems migrations, and staffing reorganizations would be carried out with great forethought, planning, and analysis. Consultants would be hired to help make plans, which would be revised, tweaked, argued over, abandoned and revived. Perhaps in the end a smooth transition would come about, but it certainly would require hard work, patience and creativity. » [4] L'entrée en vigueur rapide de la Loi 10, annoncée en décembre 2014, soit 4 mois avant la fusion, n'a pas permis aux bibliothèques du CISSS de Lanaudière de prendre le temps de planifier longuement la fusion. Diverses problématiques administratives liées au fonctionnement ont forcé le commencement de la fusion des bibliothèques dès avril 2015, bien avant qu'un projet officiel ne soit rédigé, qu'un gestionnaire ne soit assigné au projet ou que les décisions soient entérinées.

Par exemple, dès avril 2015, la bibliothèque de l'ancien CSSS du Nord de Lanaudière a été mandatée pour desservir les employés dans le secteur de la santé publique provenant de l'agence (abolie au $1^{\text {er }}$ avril 2015). La collection n'étant pas accessible (car mise en boite avant la fusion pour un déménagement) et aucun employé n'étant en poste à l'agence, la bibliothèque du CSSS du Nord de Lanaudière a dû élaborer rapidement une stratégie de récupération et une offre de service pour cette nouvelle clientèle sans que la moindre information supplémentaire ne lui soit fournie. D'autres difficultés, tels l'absence de gestionnaire et d'une bibliothécaire au CSSS du sud, rendant impossible la prise de décision pour les acquisitions ou le paiement des factures, ont aussi obligé les 2 bibliothèques des anciens CSSS à travailler en partenariat immédiatement avant que leurs rôles dans la fusion ne soient définis.

Colleen Kenefick et Jennifer A. DeVito dans leur article Five Realities of Hospital Library Mergers identifient les différents secteurs d'activités pour lesquels une planification doit être élaborée lors d'un processus de fusion: le secteur administratif, le secteur organisationnel, le service à la clientèle, l'offre de services et la gestion du personnel [5]. Si la fusion est planifiée d'avance, l'idéal est de définir des objectifs pour chacun de ces secteurs, autant en ce qui a trait au processus d'intégration qu'aux buts à atteindre.

Dès mai 2015, les 5 bibliothèques sont entrées en contact pour organiser des rencontres. Le but était de se connaître et de comprendre les réalités de chacun pour 
en venir à planifier les activités de fusion dans tous les secteurs. Les différentes actions d'intégration entreprises n'ont pas été constantes au fil des mois qui ont suivis. Parfois par essai-erreur, parfois par un travail ardu, parfois par urgence, ou encore par un processus bien défini, les bibliothèques ont dû faire preuve de flexibilité pour fusionner leurs activités.

Même si la structure organisationnelle et les procédés administratifs ont été les premiers éléments à être questionnés (organigramme, définition des rôles, utilisation des services, localisation, réalités budgétaires, mission des bibliothèques), c'est le secteur qui a été le plus long à uniformiser. Aucun de ces éléments n'a été discuté et connu au cours de 2015. Le secteur qui a demandé le plus de réactivité, a été la gestion des ressources humaines. Les coupures de postes précédant la fusion, le manque d'information sur les collections existantes et sur les nouvelles clientèles ont été suffisants pour chambarder les tâches habituelles quel que soit le niveau occupé, du gestionnaire au technicien. Sans que le projet de fusion ne soit rédigé, ils ont dû être adaptés en conséquence pour répondre aux besoins quotidiens.

Le secteur le plus exigeant à intégrer en ressources et en temps, a été la mise en commun des collections et l'uniformisation des services. Il n'était pas nécessaire de réinventer la roue, mais il fallait combiner les façons de faire et les adapter aux nouveaux besoins de l'organisation, qui étaient loin d'être connus en avril 2015. Et étant donné la diminution des ressources, il fallait aussi éliminer les services qui étaient le moins représentatif pour les utilisateurs et optimiser ceux qui l'étaient. Plusieurs étapes s'échelonnant de 2016 à 2018 ont été nécessaires pour réaliser cette partie de la fusion.

\section{Intégration de la structure organisationnelle et administrative}

«Mergers can occur for a variety of reasons, including financial pressures, hospital restructuring, new multihospital affiliations, partnering with other related departments, or changing service models. Regardless of the initial impetus for merging, there are many known issues that can be planned for, but there are also unknown issues that will arise during the merger process. » [5] La fusion des centres intégrés de santé et de services sociaux regroupe à peu près tous ces défis.

Le processus de définition de la structure organisationnelle des bibliothèques a duré plusieurs mois, surtout parce qu'il était dépendant des décisions des plus hauts niveaux de la direction. Que ce soit pour le choix de la direction, le choix du gestionnaire, le choix du responsable des bibliothèques, la définition des ressources humaines attribuées aux bibliothèques, aux budgets données et aux espaces attribués, toutes ces décisions ne pouvaient être prises par les bibliothèques, mais par les directions concernées uniquement.

Concrètement, les bibliothèques ont été informées qu'elles feraient partie de la Direction de l'enseignement universitaire et de la recherche uniquement à l'hiver 2016, soit 10 mois après la fusion, ralentissant considérablement toutes les activités de fusion envisagées. Le nouveau gestionnaire responsable des bibliothèques n'a été nommé qu'en janvier 2016, et son entrée en poste différée de plusieurs semaines. Depuis ce temps, 3 gestionnaires par intérim ont chapeauté les bibliothèques, ralentissant et alourdissant à chaque fois l'avancée des travaux de fusion. L'organigramme de la Direction a été publié officieusement en 2018 , et le directeur a été nommé uniquement en février 2019, soit 4 ans après la fusion.

Les bibliothèques ont toutefois entrepris plusieurs actions pour inspirer les décisions et faire connaitre les besoins informationnels des employés à la haute direction au cours de 2016-2016. Lumley, Datta, and Wright dans Merging and moving - the NRI Experience: an exercise in library integration and relocation [6] proposent de créer un comité de fusion pour diriger ces actions, mais comme l'équipe des bibliothèques du CISSS de Lanaudière est petite, cette solution n'a pu être retenue, et une seule personne a été désignée. Au départ, c'est la bibliothécaire de l'ancien CSSS du Nord de Lanaudière qui s'est retrouvée responsable par défaut des activités de fusion et des 5 bibliothèques de l'établissement. Son mandat a été confirmé au printemps 2016, soit un an après le début de son implication.

Être responsable des activités de fusion pour la bibliothécaire fut synonyme de responsabilités additionnelles et de prise de décisions qui n'auraient pas été de son ressort dans un autre contexte: gestion budgétaire multi-établissements et confirmation des achats pour les anciens établissements, gestion des achats groupés pour le nouvel établissement et répartition dans les anciens budgets, insertion et déménagement des collections des bibliothèques non fonctionnelles vers les bibliothèques fonctionnelles, rencontres préliminaires de tous les employés, visites de toutes les bibliothèques fusionnées et rédaction d'états de situation. Bref, une fusion est aussi synonyme de flexibilité, même à ce qui a trait aux processus décisionnels habituels. 
Le responsable de fusion doit aussi s'armer de patience. Par exemple, les budgets, les suivis budgétaires et les processus d'achats ont été unifiés uniquement en avril 2018, soit 3 ans plus tard. Un des impératifs d'un exercice de fusion est donc de prévoir un temps de transition. "Plan for time away from regular duties and from growth. Any merging of services or units will take significant time. Loss of attention, lower efficiency and less effectiveness are all possible as staff focus energy on merging. Accepting this and being open about it may waylay some morale issues. » [7]

Intégration des cultures organisationnelles et nouvelle mission

«Over time, libraries develop their own organizational history and culture that does not show up neatly on any organizational chart. Every library has its own set of unique practices that serve to bond all the participants together with a shared culture. » [8] Dès les premières rencontres entre les différents employés des bibliothèques du CISSS de Lanaudière, ces différences de culture organisationnelle se sont manifestées comme forces contraires. Juntunen, Muhonen, Nygren et Saarti l'ont souligné lors de leur exercise de fusion: « It is still somewhat difficult for the campus libraries to give up their independent character. » [9]

Même si toute l'équipe des bibliothèques s'est ralliée rapidement aux activités de fusion, plusieurs problématiques en lien avec la culture organisationnelle ont fait surface au cours des mois. Par exemple, le changement de nom des bibliothèques, les méthodes de travail et les habitudes du personnel ont suscité de nombreuses discussions. Un des défis de la fusion fut donc de prendre en compte ces différentes cultures afin d'en créer une nouvelle.

Bien sûr, chacune des bibliothèques fusionnées a dû d'abord accepter de perdre la trace de certains aspects de son identité, surtout celles qui étaient plus spécialisées. Par exemple, celle de La Myriade, devenait, malgré elle, une pièce d'un ensemble plus grand et plus global. Sa collection spécialisée, autant par son contenu que ses formats, allait être mêlée à une plus large collection identifiée à tout le CISSS.

Czechowski conseille de ne pas planifier les exercices de fusion comme des fermetures, même si c'est le cas, mais bien d'intégrer une ou plusieurs bibliothèques dans une autre [10]. C'est la direction qui a été prise par l'équipe des bibliothèques du CISSS de Lanaudière. La fusion et l'intégration à tous les niveaux des bibliothèques n'est pas un travail qui s'est fait à sens unique, où une certaine bibliothèque a pris le contrôle sur les autres. Bien au contraire, tous les partis ont pu donner leur opinion sur la planification du projet de fusion, pour ainsi en arriver à la création d'une toute nouvelle vision.

Deux rencontres de brainstorming ont été organisées à l'hiver 2017 pour permettre à toute l'équipe de se prononcer sur une nouvelle mission. «In brainstorming sessions, staff members were mixed together to enable mingling and getting acquainted. Planning work was done efficiently and at this stage, cultural clashes did not affect results in any negative way. » [11]

À la fin de cet exercice de brainstorming, il a été décidé que tous les services seraient réunis en une seule et même bibliothèque, répartis en 2 points de services, un au Centre hospitalier de Lanaudière et un à l'Hôpital Pierre-Le Gardeur. Trois énoncés de vision ont été formulés par l'équipe et plusieurs priorités d'action ont été mises de l'avant :

1. La bibliothèque est un carrefour de l'actualisation du savoir à la fine pointe des connaissances professionnelles :

- par le développement de ses collections et de ses ressources numériques,

- par le développement de veilles informationnelles et de revues de presse,

- par ses activités de diffusion.

2. La bibliothèque est un milieu accueillant, dynamique, convivial, et ressourçant :

- par son accueil chaleureux et souriant à la clientèle,

- par ses lieux conviviaux (locaux et site web) et la tranquillité,

- par l'accessibilité à ses ressources, ses services et ses lieux.

3. La bibliothèque offre un service personnalisé en fonction des besoins des clients :

- par l'offre de service des recherches spécialisées et personnalisées,

- par les formations personnalisées,

- par ses veilles informationnelles personnalisées,

- par les sondages et les outils de rétroaction offerts aux utilisateurs.

« The role of organizational values or organizational culture is a leading cause of merger success or failure. » [12] Maintenant que cet exercice est terminé, l'équipe ne peut que confirmer qu'il est très important de porter une attention particulière à cet aspect pour que la fusion réussisse. 


\section{Intégration des lieux}

Un des défis majeurs rencontrés par les bibliothèques du CISSS de Lanaudière a été d'intégrer 5 bibliothèques en 2 lieux en moins de 6 mois. Plusieurs impératifs ont obligé 3 bibliothèques (Myriade, Centre Jeunesse et Agence) à dégager les locaux qu'elles occupaient de l'automne 2015 à l'hiver 2016 pour intégrer celui des 2 autres (CSSS du Nord et du sud).

$\mathrm{La}$ planification du projet d'intégration des bibliothèques n'a pas été évident à réaliser, surtout parce que l'équipe a été pressée dans le temps. «The planning and preliminary work to merge and move a collection can be extremely exciting time or a frightening experience for staff depending on many factors. » [13] La charge de travail que ces déménagements représentaient était hors norme et contenaient une multitude d'incertitudes.

La première bibliothèque à être intégrée a été celle de l'Agence. Il fallait rapidement récupérer la collection pour pouvoir desservir les clients qui étaient maintenant sous la tutelle des autres bibliothèques. Plusieurs jours ont été nécessaires sur place pour faire l'élagage de la collection qui était trop volumineuse pour être déménagée telle quelle. Ensuite, ce fut le tour de la bibliothèque du Centre jeunesse de Lanaudière pour les mêmes raisons. À peine une journée de travail a été nécessaire pour empaqueter les livres et les revues dans des boîtes régulières. Dans les deux cas, les boîtes ont pu être entreposées temporairement dans un local près de la bibliothèque intégratrice pour que les employés puissent y accéder facilement et commencer le travail technique.

L'annonce de la troisième intégration a été plutôt surprenante, car elle était inattendue et non planifiée! La bibliothèque de La Myriade a été prévenue, à quelques semaines d'avis, qu'elle devait être déménagée étant donné le non renouvellement du bail des locaux dans lesquels elle était située. Pour réussir cette nouvelle intégration, l'équipe a dû mettre en place un processus d'élagage rapide dans les 5 collections pour dégager l'espace nécessaire pour la réaliser.

Pour chaque déménagement et intégration, l'équipe s'est inspirée des méthodes décrites par Lumley, Czechowski et Hitchcock [6, 10, 13]. En résumé, 5 étapes ont été établies pour planifier le travail :

1. Évaluation de la collection à déménager : élagage à faire, nombres de boîtes ou de bacs nécessaires pour le transport, espace nécessaire à l'arrivée pour l'entreposage, espace nécessaire pour l'intégration.
2. Empaquetage de la collection: ordre déterminé de classement dans les boîtes, identification des boîtes, scellage des boîtes.

3. Transport des boîtes: acheminement des boîtes au magasin du transport ou dans un endroit accessible aux déménageurs, suivi des boîtes durant le transport, réception des boîtes dans le nouveau lieu.

4. Entreposage temporaire des boîtes : assurer un classement temporaire des boîtes selon l'identification déterminée, assurer la possibilité d'un va-et-vient des employés venant chercher des documents pour les traiter.

5. Intégration progressive des collections : catalogage des livres, équipement des livres pour le prêt, classement sur les rayonnages, déplacement des collections existantes pour faire de la place aux nouvelles.

L'intégration totale des 3 bibliothèques et de leurs collections s'est terminée au cours de 2017.

\section{Intégration des collections}

Réunir les collections n'était pas uniquement synonyme de déménagement, il a fallu également décider, au même moment, comment serait faite l'intégration des collections entre elles et comment les données liées à ces collections seraient récupérées. L'équipe s'est arrêtée aux propos de Heron, Simpson, Weiss et Phillips [14] pour identifier les points sur lesquels s'interroger. Par exemple, devait-on uniquement déménager physiquement les collections, les placer dans des endroits distincts et conserver les anciens catalogues? Ou était-il préférable de fusionner les catalogues, d'uniformiser les notices et d'intégrer complètement les collections? Quels étaient les bénéfices et les pertes liés à ces choix? Quels étaient les impacts sur les tâches des employés et sur les services à la clientèle?

Dès le départ, il a été évident pour l'équipe que l'intégration complète des collections et l'uniformisation des données dans un seul catalogue était le meilleur choix. Premièrement, 3 des 5 collections (environ $80 \%$ des documents possédés) étaient déjà dans cette voie : même logiciel, même classification, même indexation (Appendice 1 : Tableau 2). Il ne restait donc que $20 \%$ des collections à intégrer à ces systèmes. Du point de vue des coûts, l'intégration des collections était facilement envisageable. Il faut souligner que même si les fusions sont souvent réalisées dans l'optique d'économie de ressources, que durant la 
fusion, c'est fréquemment le contraire qui se produit [4]. Malgré les coûts additionnels que cette intégration représentait, à long terme, cette solution devenait la plus économique en ressources.

Plusieurs autres facteurs ont toutefois conforté l'équipe dans cette décision. Un projet avait déjà été déposé, avant la fusion du $1^{\text {er }}$ avril 2015, par la bibliothèque du CSSS du Nord de Lanaudière pour la mise à jour du logiciel Kentika. Lui ajouter le transfert des données était simple. En choisissant de conserver ce projet, de mettre à jour le logiciel Kentika et d'y intégrer toutes les collections, il était aussi possible de bifurquer vers les nouvelles normes de catalogage RDA et adapter la classification et l'indexation aux besoins élargis du nouveau CISSS. Le catalogue Bibliofiche, qui était non accessible, dû au cloisonnement informatique, et la désuétude informatique du catalogue Regard, n'ont que confirmé ce choix. Bref, en supprimant les coûts associés au renouvellement des 4 licences annuelles sur 5 pour ces logiciels, la possibilité d'autofinancer le projet fut décisive.

\section{Intégration des données}

Heron dit: «The first stage of the Single Bib Pilot Project was to determine whether the eleven million bibliographic records of the three pilot libraries could be merged successfully without serious data loss, yet also without excessive duplicate records. » [14] Pour chaque intégration de données, l'équipe a donc évalué les données contenues dans chacun des catalogues afin de quantifier le temps nécessaire à la préparation des données, les possibilités d'erreur au transfert, les niveaux de correction des données requis après coup, et la perte des données à envisager. Bref, est-ce que le transfert valait l'investissement ou non?

La mise à jour du catalogue, ainsi que le transfert de données, donnait aussi l'opportunité à l'équipe de faire une révision des métadonnées choisies pour le nouveau catalogue «En principe, le modèle de métadonnées interne du catalogue devrait être riche au sens où il doit permettre d'y intégrer tous les degrés de complexité bibliographiques souhaités par la bibliothèque (...) » [15] Toutefois, l'équipe voulait simplifier et uniformiser la saisie future des données. Deux analyses, une sur les données existantes et une sur les métadonnées désirées pour l'avenir, ont donc été menées de front.

En pratique, les données de 3 catalogues seulement ont été transférées dans le nouveau catalogue (Appendice 1 : Tableau 2). Un simple élagage dans ces 3 collections, suivi d'une suppression stratégique de notices, de quelques changements de vocabulaire pour compléter l'adaptation aux nouvelles métadonnées, furent suffisants pour réaliser le transfert de données efficacement. Il a également été possible de repérer les doublons des différents index de façon informatique et de faire un ménage automatisé des données. Des corrections, après le transfert, ont cependant été nécessaires, principalement pour les données provenant de Regard, mais aussi pour uniformiser la classification et l'indexation en fonction des nouvelles normes. Au cours de 2016 et 2017, plus de 37000 données ont été supprimées et plus de 26000 ont été modifiés dans le nouveau catalogue pour atteindre ce but.

Les données de l'Agence, quant à elles, ont été abandonnées, car elles n'étaient plus à jour et plus de la moitié de la collection était à élaguer. Même chose pour celles du Centre jeunesse qui étaient peu nombreuses, en plus d'être non conformes du point de vue des règles de catalogage.

Ces 2 collections ont donc été complètement recataloguées, comme si elles étaient de nouvelles acquisitions, ce qui a représenté environ 1400 documents sur un total de 10000 toutes collections confondues. Une attention particulière a été apportée à la classification et à l'indexation de ces documents, afin que celles-ci reflètent bien la spécialisation de ces collections [16]. Le thésaurus préalablement importé des 3 autres catalogues a été largement bonifié afin de respecter l'unicité de ces 2 collections.

\section{Intégration des documents}

Les contraintes d'espaces, les ressources limitées consacrées à l'intégration des collections et les impératifs liés au transfert des données ont forcé un élagage massif dans les 5 collections. Il fallait éviter d'investir du temps et de l'argent sur des doublons ou des documents désuets, autant du côté des livres que des périodiques.

Tout d'abord, tous les documents disponibles en plein texte gratuitement sur le web ont été retirés des collections: publications gouvernementales et ministérielles, tables scientifiques et rapports d'organismes. Pour chaque document, 2 à 4 exemplaires ont été conservés en fonction des statistiques d'emprunt.

Au total, 9 critères d'évaluation ont été fixés pour réaliser le reste de l'élagage des documents : notoriété de l'auteur, qualité de la maison d'édition, existence de réimpression, année de publication, existence d'une nouvelle édition, rareté du document, organisation et 
qualité du contenu, collection d'appartenance, format et type de reliure.

Chaque document devait au moins répondre aux qualités de 2 critères pour être conservé. En pratique, l'équipe a utilisé l'année de publication pour débuter le travail. Tous les documents publiés avant l'an 2000, donc qui avait plus de 15 ans, ont été retirés des tablettes pour passer à l'évaluation. Pour les livres de médecine, qui sont réédités la plupart du temps à tous les 4 à 5 ans, ce critère a été abaissé à 10 ans. Pour chacun des titres retirés des tablettes, une recherche web a été effectuée pour évaluer les 8 autres critères.

Étonnamment, plusieurs documents ont pu être élagués seulement en étudiant l'auteur et la maison d'édition. Avec les années, de nombreux documents provenant d'associations et d'organismes disparus n'avaient plus du tout leur raison d'être dans la collection. Les documents n'ayant pas un format et une reliure régulière ont aussi été élagués : cahiers spirale, pamphlets, cartables, boîtes en plastique, trousses multi-supports, audiovisuels désuets. Beaucoup d'espaces et de rayonnages ont ainsi été libérés pour faire place aux collections intégrées.

\section{Intégration des acquisitions}

L'équipe des bibliothèques du CISSS a dû aussi revoir toutes les politiques et procédures d'acquisition, autant à ce qui a trait au contenu des collections qu'à leur format. Par exemple, dans les 2 CSSS, les habitudes d'acquisition étaient très larges et n'incluaient pas nécessairement les thèmes spécialisés couverts par les 3 autres bibliothèques.

En pratique, dès 2015, la liste de tous les périodiques actifs a été dressée pour chacune des bibliothèques afin de suivre de près les renouvellements. Pour commencer, il s'agissait de canceller les doublons, pour pallier aux contraintes budgétaires et d'espace [6]. Pour les périodiques imprimés, les renouvellements et annulations ont été aisés. Cependant, pour toutes les publications numériques, il a fallu négocier férocement avec les éditeurs et les distributeurs pour éviter que le prix augmente drastiquement en fonction de la grosseur de la nouvelle organisation. Certains abonnements numériques ont dû être abandonnés. Par exemple, dans le cas du New England Journal of Medicine, le prix était 5 fois plus élevé pour la nouvelle organisation que pour celle qui l'avait pris l'année précédant la fusion.

D'un autre côté, il a été possible d'envisager un virage numérique pour plusieurs titres. Comme la nouvelle organisation comptait désormais plus de 10000 employés déployés dans 61 établissements sur un large territoire, se diriger vers cette voie devenait impératif pour assurer un accès égalitaire aux collections. Les abonnements imprimés ont été progressivement transférés en numérique au cours de 2016 et 2017. Au final, en 2018, seulement 5\% de la collection de périodique était encore en format imprimé.

En 2017, une collection de livres numériques a aussi fait son entrée dans le catalogue, modifiant les politiques d'acquisition antérieures qui faisaient uniquement référence à des livres imprimés. Depuis, environ $30 \%$ du budget d'acquisition des livres est désormais consacrés aux livres numériques chaque année.

Aujourd'hui, la collection compte plus de 12000 documents en santé (médecine générale, médecine spécialisée, soins infirmiers, professions multidisciplinaires), services sociaux, psychologie, informatique, gestion et administration sous différents formats : livres imprimés, livres numériques, documents audiovisuels, revues d'intérêt général, revues spécialisées imprimées et en ligne, bases de données spécialisées.

\section{Intégration de l'offre de services}

«There must be some sense of perceived gain to contemplate a merger, and improving services is the ultimate gain. This is a perfect time to ask users what they truly expect of library services so that services can then reflect their needs. » [5] Saisir, au fil des échanges avec ses clients, les diverses problématiques à régler et les nouveaux enjeux engendrés par la fusion de l'établissement, a permis aux bibliothèques de réévaluer l'offre de services.

Avant le $1^{\text {er }}$ avril 2015, toutes les bibliothèques offraient des services de proximité aux clients. Toutes avaient un catalogue consultable sur l'intranet et proposaient leurs services de façon traditionnelle dans les locaux de la bibliothèque. Il y avait bien sûr des abonnements en ligne, mais ils étaient principalement utilisés par les membres de l'équipe des bibliothèques et transférés aux clients sur demande. Avec la fusion, très rapidement, les outils et les services de proximité existants sont devenus inadaptés à la demande.

Il est rapidement apparu évident que la nouvelle bibliothèque intégrée devait se doter d'outils, de collections et de services tous accessibles à distance, pouvant être consultés, demandés et reçus par les 10000 employés dans les 61 établissements dans les meilleurs délais possibles. Donc, sans réellement changer l'essence des services des bibliothèques qui 
sont restés à la base les mêmes (le catalogue en ligne, le prêt de document, le prêt entre bibliothèques, les recherches spécialisées, et les veilles informationnelles), l'intégration des services a forcé le passage vers les outils en ligne. "Another factor in reorganizing was to be able to offer new services by aligning staff in more efficient and synergistic ways. » [17] Faire le virage numérique et ainsi, minimiser les anciennes limites imposées par les services de proximité, a été le cheval de bataille de l'intégration des services.

\section{Intégration des services web}

Avant le $1^{\text {er }}$ avril 2015, les clients consultaient leur catalogue sur le site intranet de leur organisation. Durant la transition, l'équipe a dû multiplier les recherches dans 5 catalogues pour répondre à une simple requête du genre «Avez-vous ce livre? ». Une des priorités suite à l'intégration des collections et des catalogues a donc été de développer le site web de la nouvelle bibliothèque et de rendre le catalogue unifié, ainsi que les autres services, disponibles en ligne à un seul et même endroit. Soit, à peine 4 mois après l'intégration des données dans le nouveau catalogue (et 2 ans après la fusion), c'est-à-dire en avril 2017, le site web a été lancé.

Le changement était majeur autant pour l'équipe que pour les clients. De 5 catalogues utilisant des plateformes différentes accessibles sur 5 intranets, nous sommes passés à un seul catalogue accessible via un seul site web disponible sur internet: http://bibliocissslanaudiere.visard.ca. Les anciens catalogues comptaient moins de 1000 recherches mensuellement. Dans les mois suivant la diffusion du nouveau site web, les recherches dans le catalogue unifié sont grimpées entre 35000 et 50000 par mois, pour se stabiliser entre 10000 et 20000 au cours de 2018. Signe de réussite, le travail de formation et de soutien auprès des clients pour en arriver là a toutefois été colossal.

Plusieurs autres volets des services de la bibliothèque ont également été déployés sur le site web afin d'en faciliter l'accès : les dossiers thématiques, les nouveautés, et les ressources en ligne. «Providing a portal via the catalog was perhaps the easiest step in expanding access to electronic resources (...) ». [4]

Une adresse email unique a aussi été créée pour toutes les demandes arrivant et sortant du catalogue et du site web. Juntunen, Muhonen, Nygrén, et Saarti affirment que la création d'un email commun, tout comme l'intégration des services, est nécessaire : «Its aim is to create unified, clear, and functional client services in all HU libraries so that clients get welldefined and organized services, which fulfil their needs at all campuses as well as from the Internet. » [9] Des modèles de courriel ont même été développés afin de répondre aux questions les plus courantes : comment s'abonner à la bibliothèque, quels sont les services offerts, comment s'inscrire aux veilles informationnelles, ou bénéficier d'une recherche spécialisée.

\section{Intégration du prêt de document}

Malgré tous les efforts déployés dans le développement des services en ligne, certains services, tel le prêt de document imprimé, ne pouvaient se dématérialiser autant que les autres. Un service de prêt à distance a donc été développé pour cette collection. Une procédure de demande en ligne pour le prêt a été instaurée dans le catalogue pour faciliter la vie aux utilisateurs et la réception des demandes. Comme les documents se trouvaient aussi désormais dans 2 points de services différents, un nouveau système de communication a dû être instauré entre les membres de l'équipe responsable du prêt à distance pour s'assurer de la circulation efficace des documents.

Pour minimiser les efforts induits par le prêt de documents imprimés à distance, les collections numériques ont été développé, via les périodiques comme il a été expliqué précédemment, mais aussi avec l'ajout d'un tout nouveau volet à la collection : les livres numériques. Plus de 300 livres numériques sont maintenant disponibles dans le catalogue.

\section{Intégration des veilles informationnelles}

Pendant les premiers mois de la fusion, chacune des bibliothèques a continué les veilles telles quelles. Les veilles étaient trop diverses dans leurs formes et leurs contenus pour qu'elles puissent être fusionnées rapidement. Le problème principal était que les processus de veille comportaient de longues interventions manuelles. Par exemple, plusieurs veilles comprenaient la numérisation d'une table des matières, un envoi de courriel par titre ou par sujet, à une liste de clients précis. Vu le nombre de titres de périodiques une fois les collections regroupées et le nombre d'employés total de l'établissement, il était impossible de continuer dans cette voie.

« Throughout the entire merger process, it was the intention that services not be negatively affected and that, whenever possible, services be identical across all libraries, keeping in mind the special requirements of 
various users groups. » [8] Les objectifs étaient de trouver une forme de veille qui allait répondre aux divers intérêts des clients, tout en rendant le processus plus léger pour l'équipe, et étaient aussi d'optimiser les outils pour assimiler la hausse des inscriptions. Les outils du nouveau catalogue ont été choisis pour développer la nouvelle offre de service de veilles informationnelles, pour conserver un maximum d'uniformisation dans les services en ligne.

Tout d'abord, à l'automne 2017, des dossiers thématiques ont été créés. Chaque dossier est une requête de recherche automatisée. Elle rapporte tous les documents sur un sujet donné se trouvant dans le catalogue. À partir de ces dossiers thématiques, des veilles informationnelles sur les nouveautés entrées au catalogue, sont envoyées à chaque mois par courriel. Chaque client abonné à la bibliothèque est automatiquement inscrit aux dossiers thématiques et reçoit les courriels de nouveautés. Comme l'inscription se fait par thématique ciblée, chaque client ne reçoit que l'information qui est pertinente pour lui. Et comme il est possible de créer un nombre de dossiers thématiques infinis, les veilles peuvent être tout autant générales que spécifiques et se développer au fil des besoins. D'une centaine de veilles nouveautés envoyées par mois, la bibliothèque en envoie plus de 1000 aujourd'hui, sans que la charge de travail ne soit augmentée.

Dans un deuxième temps, les veilles informationnelles en lien avec les périodiques ont été développées. Le nouveau catalogue proposait un lecteur de flux RSS sur lequel il était possible de construire des veilles thématiques. Le même principe que pour les dossiers thématiques a été appliqué pour la veille des flux RSS : création de thématiques, enregistrement de flux RSS dans la requête automatisée et inscription des utilisateurs selon leurs intérêts. De plus, la transformation progressive de la collection de périodiques en numérique, a permis de fournir aux clients directement dans le bulletin de veille, le lien vers l'article pour qu'ils puissent le télécharger eux-mêmes. De la possibilité de desservir entre 100 et 200 clients mensuellement, nous sommes passés à plus de 1500 . L'optimisation de ces services permettra de développer au fil du temps assez de thématiques pour satisfaire le plus grand nombre de clients possible.

\section{Intégration des clients}

«The transition from brick-and-mortar book repositories to virtual services and collaborative spaces is most challenging to small libraries. » [17] Autant pour l'équipe que pour les clients, ces nouveaux services à distance et numérique furent déstabilisants. Un plan de communication, incluant des capsules de formation, a donc été développé pour informer et former les clients, aux services intégrés de la nouvelle bibliothèque fusionnée.

Tout d'abord, l'annonce du déploiement du site web et du catalogue en ligne a été publiée sur l'intranet organisationnel. Un lien permanent vers le site est aussi disponible à partir d'un onglet bien visible. Le même onglet est aussi disponible sur le site internet de l'organisation pour les clients consultant à l'externe.

Des articles informatifs sur les différents services ont aussi été publiés de façon régulière et continue dans les publications officielles de l'organisation [18]. Un visuel d'identification propre à la bibliothèque du CISSS de Lanaudière est utilisé pour toutes les publications. Quelques pages Facebook organisationnelles ont également été ciblées pour diffuser la même information. Un signet imprimé, contenant les coordonnées et services de la bibliothèque, a aussi été distribué.

De plus, un courriel formatif a été envoyé à tous les clients inscrits à la bibliothèque mensuellement. Chacun de ces courriels comprenait la procédure pour utiliser un des services en ligne. L'information transmise aux utilisateurs et les formations qui en ont découlé visait à faciliter la transition, autant du point de vue des membres de l'équipe de la bibliothèque que des clients.

\section{Intégration du personnel}

«Perhaps the most difficult merger problems to deal with are staffing and personnel. Staffing issues at multiple sites are not easily known at the beginning, and they will be issues long after a merger is officially complete. Success or failure of a partnership or merger largely be determined by personalities, working relationships, and institutional histories. » [5] La fusion des bibliothèques, tout comme celle de l'établissement, a occasionné des coupures et des mouvements de personnel, laissant certaines bibliothèques sans voix, créant un climat d'incertitude dans l'équipe. Dès décembre 2015, un premier membre de l'équipe a quitté pour la retraite. En 2016, 2 autres ont été transférés à temps plein dans l'équipe de la gestion documentaire.

Ces changements organisationnels ont affecté grandement les ressources humaines : perte d'identité, diminution du sentiment de sécurité, obsession pour la survie même, perte de bons employés et perte d'estime de soi. [12] L'équipe des bibliothèques n'a pas fait exception à la règle. 


\section{Intégration de la communication}

Dès avril 2015, la responsable de la fusion des bibliothèques a organisé plusieurs rencontres informelles entre les différentes bibliothèques pour permettre à l'équipe d'apprendre à se connaître et pour prendre connaissance de l'état de situation. Lorsque le gestionnaire a été nommé à l'hiver 2016, il a été possible de créer un calendrier des réunions mensuelles pour que l'équipe puisse entrer en contact régulièrement. "Staff members need to be given opportunities to voice their opinions and recommendations for improvements even if they do not have the authority to alter final decisions. By assisting in setting joint goals, they will be more invested in the process and have more ownership in seeing these goals succeed. » [5] Les réunions mensuelles ont servi à tenir les membres de l'équipe informés sur les activités d'intégration et de lieu de parole.

Malgré un processus de communication officiel et des milliers d'ajustements réalisés après chacune de ses réunions, le niveau de stress était parfois important dans l'équipe. Plusieurs lenteurs administratives ont retardé le dénouement de problématiques importantes. Plusieurs frustrations relevaient aussi des différentes personnalités et de la capacité de chacun à lâcher prise sur les anciennes façons de faire.

Dans tous les cas, tous les projets d'intégration, ont été réalisés avec succès, mais pour certains, le mécontentement a été palpable pendant plusieurs semaines. Pour faire face à ces tensions, Stephenson suggère de rappeler à tous, que chacun des membres de l'équipe a des intentions positives envers le projet et qu'il est préférable de focusser sur les forces que sur les faiblesses [19].

\section{Intégration des tâches}

Dès avril 2015, certaines tâches ont été révisées pour tous les membres de l'équipe. L'impact a été minime au départ. Il s'agissait surtout d'éliminer les tâches effectuées en double. Par exemple, les acquisitions et les achats ont été expressément remis entre les mains de la bibliothécaire, et la gestion de tous les périodiques remises à une seule et même technicienne.

Toutefois, au cours de 2016-2017, les ajustements ont été plus difficiles. Même si les services sont restée à la base les mêmes, le développement des services en ligne et à distance a modifié pratiquement tous les processus reliés aux tâches. "Working in a new, merged library has been challenging for all library staff members. The amount of learning and adoption of new policies and procedures required within the merged library in and of itself would have been more than enough to tax staff. » [9]

Chaque aspect du projet de fusion mis en place en 2016-2017 était synonyme d'un nouvel apprentissage pour les membres de l'équipe. De nombreuses formations ont été données, de nombreuses procédures ont été rédigées pour mettre de l'ordre dans ces nouveaux processus, mais malgré tout, l'équipe a dû faire face à beaucoup d'incertitude et d'inconnu. Bref, il faut prévoir un temps d'adaptation à chaque étape et reconnaître que les changements influenceront l'efficacité de l'équipe. Il faut surtout accepter le fait que l'apparition de tensions est inévitable et savoir écouter les ressentis de chacun.

\section{Conclusion}

Les divers auteurs ayant étudié et décrit des fusions de bibliothèques concluent souvent leurs articles en parlant des différents gains obtenus par ce changement. Il va sans dire que les avantages et les progrès réalisés aux cours de la fusion des bibliothèques du CISSS de Lanaudière sont nombreux. La fusion a permis à l'équipe de développer un catalogue au goût du jour, de créer un site web intéressant pour les clients, de partager des collections, d'accroître les services en ligne, et surtout d'améliorer grandement son offre de services aux utilisateurs. Les difficultés à surmonter ont été innombrables, tant au niveau des ressources disponibles qu'aux difficultés d'adaptation rencontrés par les différents membres de l'équipe. Néanmoins, au final l'expérience s'avère positive. Les clients sont satisfaits et félicitent régulièrement l'équipe des services qu'ils reçoivent.

\section{Statement of Competing Interests}

No competing interests declared.

\section{References}

1. Assemblée nationale du Québec. Loi modifiant l'organisation et la gouvernance du réseau de la santé et des services sociaux notamment par l'abolition des agences régionales [Internet]. Québec; 2015. Disponible au : http://www2.publicationsduquebec.gouv.qc.ca/dyn amicSearch/telecharge.php?type $=5 \&$ file $=2015 \mathrm{C} 1 \mathrm{~F}$. PDF 
2. Ministère de la santé et des services sociaux. Projet de loi modifiant l'organisation et la gouvernance du réseau de la santé et des services sociaux notamment par l'abolition des agences régionales [Internet]. Québec; 2014. Disponible au : http://www.msss.gouv.qc.ca/documentation/sallede-presse/medias/Presentation-infotechniquePL10.pdf

3. Centre intégré de santé et de services sociaux de Lanaudière. Info-CISSS de Lanaudière. 2015; 1(3) $: 1-4$.

4. Rozum B, Brassaw L. Merging two academic libraries: finding unity from diversity while maintaining institutional identities. Adv Librariansh. 2013; 37: 201-211.

5. Kenefick C, DeVito JA. 2015. Five realities of hospital library mergers. J Hosp Librariansh. 2015; 15(3):334-340.

6. Lumley A, Datta V.K, Wright J.A. Merging and moving - the NRI experience: an exercise in library integration and relocation. Aslib Proc. 1991; 43(4):115-132.

7. Anderson, SS. Bigger than the both of us: considerations before partnering and merging, Libr Issues. 2014; 34(3).

8. Barnes I. Special libraries, special challenges. Public Serv Q. 2013; 9: 259-265.

9. Juntunen A, Muhonen A, Nygren U, Saarti J. Reinventing the academic library and its mission: service design in three merged finnish libraries. Adv Librariansh. 2013; 36:225-245.

10.Czechowski L, Barger R, Fort M, Maxeiner G. Letting go: closing a branch library of the health sciences library system, University of Pittsburgh, Libr Resour Tech Serv. 2009; 54(3):153-163.
11. Muhonen A, Nygren U, Saarti J. Bringing order out of chaos: benchmarking tools used in merging university libraries in Finland. Adv Librariansh. 2011; 34:183-205.

12. Moralo, A. Merging large and complex library organizations. Adv Libr Adm Organ. 2014; 32:265306.

13. Hitchcock M, Sager R, Schneider J. And then there was one: moving and merging three health science library collections, Issues Science Technol Librariansh. 2005; 44.

14.Heron, SJ, Simpson B, Weiss AK, Phillips J. Merging catalogs: creating a shared bibliographic environment for the state university libraries of Florida, Cat Classif Q. 2013; 51:139-155.

15.Lemaire A. Les métadonnées et les bibliothèques. Dans: Galaup X, directeur. Informatiser la gestion de sa bibliothèque. Paris: Association des Bibliothécaires de France; 2016. p. 37-54.

16. Moragas M. Réunion des fonds spécialisés à la Bibliothèque Gabriel Ferraté - Catalogne, No 1: Les topographies du savoir, Bull bibli France [Internet]. 2001. Disponible au : http://bbf.enssib.fr/consulter/bbf-2001-01-0079-009

17.Doherty B, Piper A. Creating a new organizational structure for a small academic library: the merging of technical services and access services. Tech Serv Q. 2015; 32:160-172.

18. Hanergie MP. Penetrating the hospital environment and promoting library resources, J Hosp Librariansh. 2015; 15: 217-225.

19. Stephenson K. Sharing control, embracing collaboration: cross-campus partnerships for library website design and management. J Electron Resour Librariansh. 2012; 24:91-100. 
Appendice A

Tableau 1 : Portrait des établissements fusionnés au $1^{\text {er }}$ avril 2015 et de leurs bibliothèques

\begin{tabular}{|c|c|c|c|c|c|c|}
\hline Établissements & Installations & Employés & $\begin{array}{l}\text { Personnel de la } \\
\text { bibliothèque }\end{array}$ & $\begin{array}{l}\text { Contenu de la } \\
\text { collection }\end{array}$ & $\begin{array}{l}\text { Type de } \\
\text { documents }\end{array}$ & Forces et faiblesses \\
\hline CSSS du Nord de Lanaudière & $\begin{array}{l}1 \text { hôpital } \\
9 \text { centres locaux de soins communautaires } \\
11 \text { centres d'hébergement } \\
1 \text { centre réadaptation en dépendance } \\
2 \text { services externes de psychiatrie } \\
1 \text { clinique de médecine familiale }\end{array}$ & $\begin{array}{l}5000 \text { employés } \\
350 \text { médecins }\end{array}$ & $\begin{array}{l}1 \text { bibliothécaire } \\
1.6 \text { techniciens en } \\
\text { documentation }\end{array}$ & $\begin{array}{l}\text { Médecine } \\
\text { Soins infirmiers } \\
\text { Psychologie } \\
\text { Gestion } \\
\text { Informatique } \\
\text { Services sociaux } \\
\text { Administration } \\
\end{array}$ & $\begin{array}{l}\text { Imprimé } \\
\text { Numérique } \\
\text { Audiovisuel }\end{array}$ & $\begin{array}{l}\text { Grands espaces bien aménagés } \\
\text { Locaux nouvellement réaménagés } \\
\text { Budget d'acquisition adapté aux réalités } \\
\text { Centrale dans la région nord } \\
\text { Personnel expérimenté } \\
\text { Collection à jour }\end{array}$ \\
\hline CSSS du Sud de Lanaudière & $\begin{array}{l}1 \text { hôpital } \\
2 \text { centres locaux de soins communautaires } \\
3 \text { centres d'hébergement } \\
1 \text { service à l'enfance } \\
1 \text { clinique de médecine familiale }\end{array}$ & $\begin{array}{l}4000 \text { employés } \\
380 \text { médecins }\end{array}$ & $\begin{array}{l}2 \text { techniciens en } \\
\text { documentation }\end{array}$ & $\begin{array}{l}\text { Médecine } \\
\text { Soins infirmiers } \\
\text { Ouvrages de } \\
\text { vulgarisation pour } \\
\text { patients }\end{array}$ & $\begin{array}{l}\text { Imprimé } \\
\text { Numérique } \\
\text { Audiovisuel }\end{array}$ & $\begin{array}{l}\text { Locaux nouvellement aménagés } \\
\text { Centrale dans la région sud } \\
\text { Bibliothécaire non remplacée depuis } 2 \text { ans } \\
\text { Coupure partielle du budget d'acquisition avant } \\
\text { fusion } \\
\text { Nouveau personnel } \\
\text { Collection partiellement à jour }\end{array}$ \\
\hline $\begin{array}{l}\text { Agence de santé et de services } \\
\text { sociaux de Lanaudière }\end{array}$ & 1 centre administratif & 200 employés & 0 & $\begin{array}{l}\text { Santé publique } \\
\text { Publications } \\
\text { gouvernementales }\end{array}$ & Imprimé & $\begin{array}{l}\text { Coupure } 1 \text { poste technicien avant fusion } \\
\text { Coupure du budget d'acquisition } \\
\text { Coupure des services } \\
\text { Collection non à jour } \\
\end{array}$ \\
\hline $\begin{array}{lcc}\text { Centre de } & \text { réadaptation } \\
\text { déficience } & \text { physique } & \text { Le } \\
\text { Bouclier } & & \\
\end{array}$ & 6 centres locaux de services & $\begin{array}{l}250 \text { employés } \\
7 \text { médecins }\end{array}$ & 0 & 0 & 0 & $\begin{array}{l}\text { Bibliothèque intégrée dans un autre CISSS } \\
\text { Aucune ressource }\end{array}$ \\
\hline \begin{tabular}{lcc|} 
Centre de réadaptation & en \\
déficience intellectuelle & La \\
Myriade & &
\end{tabular} & 10 centres locaux de services & 400 employés & $\begin{array}{l}0.6 \text { technicienne en } \\
\text { documentation }\end{array}$ & $\begin{array}{l}\text { Déficience } \\
\text { intellectuelle } \\
\text { Autisme }\end{array}$ & $\begin{array}{l}\text { Imprimé } \\
\text { Audiovisuel }\end{array}$ & $\begin{array}{l}\text { Coupure } 0.4 \text { technicien avant fusion } \\
\text { Coupure total du budget d'acquisition avant } \\
\text { fusion } \\
\text { Locaux dispersés et inadaptés } \\
\text { Collection petite partiellement à jour }\end{array}$ \\
\hline Centres jeunesse de Lanaudière & $\begin{array}{l}5 \text { centres locaux de services } \\
2 \text { foyers de groupe }\end{array}$ & 630 employés & 0 & $\begin{array}{l}\text { Protection } \\
\text { l'enfance }\end{array}$ & Imprimé & $\begin{array}{l}\text { Budget d'acquisition minime } \\
\text { Aucun espace dédié } \\
\text { Aucun personnel dédié } \\
\text { Collection petite partiellement à jour }\end{array}$ \\
\hline
\end{tabular}


Tableau 2 : Portraits des catalogues des bibliothèques fusionnées au $1^{\text {er }}$ avril 2015

\begin{tabular}{|c|c|c|c|c|c|}
\hline Bibliothèques & Logiciel & Classification & Indexation & État & $\begin{array}{l}\text { Transfert des données } \\
\text { vers le nouveau catalogue }\end{array}$ \\
\hline CSSS du Nord & Kentika & RCAA2 & $\begin{array}{lr}\text { Répertoire } & \text { Vedette- } \\
\text { Matière } & \text { Université } \\
\text { Laval } & \end{array}$ & $\grave{A}$ jour & Oui \\
\hline$\overline{C S S S}$ du Sud & Kentika & RCAA2 & $\begin{array}{lr}\text { Répertoire } & \text { Vedette- } \\
\text { Matière } & \text { Université } \\
\text { Laval } & \end{array}$ & $\grave{A}$ jour & Oui \\
\hline Agence & Kentika & RCAA2 & $\begin{array}{lr}\text { Répertoire } & \text { Vedette- } \\
\text { Matière } & \text { Université } \\
\text { Laval } & \end{array}$ & Non à jour & Non \\
\hline La Myriade & Regard & Dewey & Thésaurus spécialisé & À jour & Oui \\
\hline $\begin{array}{l}\text { Centre } \\
\text { jeunesse }\end{array}$ & Bibliofiche & Libre & Vocabulaire libre & Non à jour & Non \\
\hline
\end{tabular}

